Voix et Images

voixetimages

\title{
Étude de la structure anaphorique dans la Montagne secrète de Gabrielle Roy
}

\section{Marie Grenier-Francoeur}

Volume 1, numéro 3, avril 1976

Gérard Bessette

URI : https://id.erudit.org/iderudit/200039ar

DOI : https://doi.org/10.7202/200039ar

Aller au sommaire du numéro

Éditeur(s)

Les Presses de l'Université du Québec

ISSN

0318-9201 (imprimé)

1705-933X (numérique)

Découvrir la revue

Citer cet article

Grenier-Francoeur, M. (1976). Étude de la structure anaphorique dans la Montagne secrète de Gabrielle Roy. Voix et Images, 1(3), 387-405.

https://doi.org/10.7202/200039ar d'utilisation que vous pouvez consulter en ligne.

https://apropos.erudit.org/fr/usagers/politique-dutilisation/ 


\section{Étude de la structure anaphorique dans la Montagne secrète de Gabrielle Roy}

Parties intégrantes de ce que nous sommes convenus maintenant d'appeler le récit comme discours, les préfigurations littéraires sont à proprement parler des signes anaphoriques, c'est-à-dire des éléments à fonction essentiellement organisatrice et cohésive qui constituent autant de relais dans le système-mémoire complexe de l'œuvre littéraire. C'est précisément, comme l'écrit Philippe Hamon, par un tel réseau de signes anaphoriques que "l'œuvre se cite elle-même et se construit comme tautologique ${ }^{1}$.

Le roman de Gabrielle Roy, la Montagne secrète, objet de la présente étude, se signale par la richesse de son système préfiguratif. L'œuvre développe successivement deux réseaux mythologiques. Le premier, externe et conventionnel, est fondé, comme c'est la règle, sur une suite d'allusions à certains mythes anciens, à d'autres, contemporains ou presque, et sur des citations d'cuvres littéraires. Le second, issu de la trame même du roman, est donc interne et essentiellement métaphorique.

C'est en appliquant à l'œuvre de la romancière québécoise les principes d'analyse mythocritique exposés par J. J. White dans son essai, Mythology in the Modern Novel ${ }^{2}$, que nous sommes parvenue à dégager le premier réseau mythologique. Pour le second, il nous a fallu forger notre propre instrument de travail car l'auteur de Mythology in the Modern Novel ne s'attachait qu'à décrire le fonctionnement des mythes anciens, des légendes, des œuvres littéraires du passé comme préfigurations, donc comme principe structurant de nombreux romans du vingtième siècle écrits en langue allemande et anglaise surtout. Notre intérêt, il convient de le souligner, ne porte pas tant sur le mythe lui-même que sur les rapports qui l'unissent à l'œuvre particulière où il s'insère et forme un ou plusieurs motifs mythologiques.

Généralement un motif mythologique s'élabore en une chaîne d'allusions mythologiques interdépendantes habilement distribuées à des points marqués tout au long du récit. On reconnaît là la marque des signes anaphoriques qui «tissent dans l'énoncé un réseau d'appels et de rappels à des segments d'énoncés disjoints et de longueur variable ${ }^{3}$ " comme l'écrit 
si justement Philippe Hamon à propos des personnages-anaphores. La chaîne des allusions, et dans certains cas des citations, s'organise en un dessin ou pattern cohérent qu'il est essentiel au lecteur de saisir s'il veut décoder l'œuvre. Cependant il arrive que la seule mention d'un nom mythologique dans le titre d'un roman suffise à créer un motif. Toute l'analogie manifestée par la présence de ce nom à caractère mythologique dans le titre se développe alors sur le mode implicite. Mais implicite ou longuement élaborée en motif par un tissu de citations et d'allusions, c'est toujours essentiellement l'analogie entre le mythe et la trame des événements contemporains décrits dans le román qui structure ce dernier.

L'introduction d'un motif mythologique dans un roman peut se faire, en tout premier lieu, grâce au titre. Agissant à la façon d'un index pointé vers le texte, le titre indique ce qui le suit et le désigne dans son contenu. Dans le cas qui nous occupe, le titre, la Montagne secrète, nous révèle le but des longues errances du héros, l'objet de sa quête. Le mot est lâché: nous sommes forcés d'en convenir, une lecture même superficielle permet à tout le monde de constater que la trame du récit s'organise à la façon d'un mythe de la quête. Cela ne mène pas très loin car l'expression a mythe de la quête» ne désigne pas un mythe en particulier mais un ensemble paradigmatique de mythes qui englobe tout aussi bien les errances d'Ulysse que l'expédition de Jason et des Argonautes, la quête du Graal que les aventures de n'importe quel héros folklorique du type que Propp appelle justement héros-quêteurs ${ }^{4}$.

J. J. White, par un souci de rigueur que nous avons voulu faire nôtre, s'interdit de parler d'un mythe en particulier tant qu'il n'a pu relever de façon certaine ses traces dans un texte donné. Nous nous interdirons donc désormais l'utilisation de l'expression "mythe de la quête" tant et aussi longtemps que nous n'aurons isolé et iḍentifié l'un de ces mythes de la quête auxquels le titre semble faire allusion.

Cela ne signifie pas que nous rejetons cette idée de quête qui s'impose d'emblée au lecteur. Nous en faisons pour l'instant une hypothèse de travail qui devra se vérifier dans le processus analytique. S'il y a quête, il nous semble utile voire nécessaire de décomposer cette dernière en ses trois étapes obligées: l'étape préliminaire ou vocation, l'étape d'accomplissement du processus ou la quête proprement dite, et la troisième étape, le but atteint, la mission accomplie. A chacune des étapes devrait correspondre un substrat, l'archétype, couche profonde de l'œuvre, sorte d'humus secret et qui le demeurerait si les mythes dans lesquels il s'incarne ne trahissaient sa présence.

Une des premières scènes du roman, l'arrivée de Pierre Cadorai en canot par la rivière, arrivée qui se présente comme une réponse à la longue attente du vieux Gédéon, le solitaire, nous fournit quelques indices quant à la nature de l'archétype qui régit la première phase du processus, l'Enfant divin. 
Gédéon, le chercheur d'or, c'est l'attente personnifiée, le prisonnier de l'espérance. Livré à la solitude "quelque part dans les Territoires du Nord-Ouest ${ }^{5}$ " depuis la mort de sa femme et le départ de sa fille Nina, le vieil homme s'abandonne corps et âme à la fascination d'un espoir insensé. If n'ose partir vers un ailleurs plus prometteur car, sait-on jamais, peut-être la rivière après avoir longtemps éprouvé sa constance, finiraitelle demain, aujourd'hui même, par rouler vers lui ses pépites d'or, récompense de sa longue patience. Aux peines de l'isolement s'ajoute, encore plus pénible, la souffrance morale. N'est-il pas devenu aux yeux des rares passants “ une curiosité, le dernier homme, paraît-il, à chercher de l'or dans les galets d'une rivière ${ }^{6}$. L'arrivée de Pierre se produit à un moment crucial dans la vie du vieil homme: elle se présente comme un signe du destin, une réponse providentielle au solitaire qui commence à souffrir du mal qui le fera sombrer dans la folie. Entendant les coups réguliers de la pagaie du héros frappant la rivière, “Gédéon s'aperçut que l'avait effleuré ce doute monstrueux: ni en ce ciel lointain ni sur cette terre lointaine il n'existait de pensée qui se préoccupât de lui ${ }^{7}$ ". La venue de Pierre comble un moment l'attente de l'homme et lui redonne espoir. Gédéon va même jusqu'à proposer à Pierre de s'associer à lui. Mais le visiteur inconnu a mieux à offrir au vieillard. Il dessine son portrait et dans la révélation soudaine, dans le choc qu'éprouve le chercheur d'or lorsqu'il se reconnaît dans le portrait, il y a le germe d'une libération de son destin. Quand on reconquiert son image, on reprend possession de son âme. C'est un des principes de la pensée magique ou mythique propre aux primitifs, dont les rites vaudou nous fournissent un exemple. Dans l'optique de la pensée mythique qui régit d'ailleurs la vie inconsciente des primitifs comme des civilisés, le héros se présente comme un libérateur, un « messie ${ }^{8}$ ».

Deux motifs mythologiques secondaires font référence à cet archétype de l'Enfant divin et s'inscrivent ainsi dans la première phase du processus de la quête, l'appel ou la vocation à la quête. Il s'agit du motif du "Buisson ardent" et de celui de la "Lutte avec l'ange". Les deux motifs sont d'origine biblique et, fait intéressant à signaler, l'exégèse chrétienne voit en Moïse et en Jacob deux préfigurations du Messie. Pour nous, il importe de nous souvenir que le récit de l'épisode du Buisson ardent constitue dans la vie de Moïse la révélation de la vocation de ce dernier à devenir le chef inspiré du peuple d'Israël ${ }^{9}$. Cette révélation se produit au terme d'une longue errance de Moïse dans le désert alors qu'il gardait les troupeaux de son beau-père. Dans le roman de Gabrielle Roy, le héros découvre sa Montagne au terme de ses voyages nordiques, après avoir connu des périls et des avanies de toutes sortes, après avoir connu des moments d'exaltation et des moments de désespérance, après avoir résisté au désir légitime «d'être parmi des hommes, un homme simplement ${ }^{10}$ ». Le passage du roman qui fait référence au récit biblique du Buisson ardent se lit comme suit :

II se leva d'un élan brusque, avant que ne lui en fût ravie l'impulsion. II prit son canot, le souleva au-dessus de sa tête, remonta suivre le rebord périlleux du roc. Toute la paroi était à présent assombrie, le 
soleil ayant décliné derrière elle. Mais il devait luire non loin dans une échancrure profonde. Pierre voyait grandir devant lui une tache lumineuse. Il fit quelques pas encore, tourna le flanc sombre du rocher. Devant lui se dressait une haute montagne isolée que le soleil rouge embrasait et faisait brûler comme un grand feu clair.

Pierre, d'un coup d'épaule se débarrassa du canot, se défit de son sac, se laissa lui-même tomber comme à genoux devant la montagne ${ }^{11}$.

Les rapports de Pierre avec sa Montagne seront ceux du dévôt avec son dieu. Et tout au long du roman, la Montagne conservera ses traits de Jéhovah ombrageux et dispensateur de châtiments redoutables. Pierre qui entretient avec toute la nature des rapports inspirés d'une pensée animiste fait lui-même de sa Montagne une divinité anthropomorphe. Le narrateur, se faisant écho du héros et rendant ainsi encore plus manifeste le mouvement de la pensée mythique qui sous-tend cet univers romanesque, décrit de la façon suivante la Montagne à l'approche de l'hiver: "La montagne s'obscurcit comme un vaste front chargé d'inquiétude ${ }^{12}$."

Orok, le chasseur esquimau que Pierre rencontre au pied de la Montagne reconnaît qu'à “cet homme Dieu devait parler mieux qu'à Orok ${ }^{13 \text { ". }}$ Mais il n'est en aucune façon envieux de ce privilège.conféré à celui qu'il appelle l'Homme-au-crayon-magique. Il sait que ce n'est pas «toujours souhaitable d'être celui à qui Dieu parle. Ne s'expliquant pas nécessairement avec clarté, Dieu était néanmoins mécontent de n'être pas compris ${ }^{14}$ ". Le dernier conseil qu'Orok lance à Pierre avant de s'éloigner nous confirme dans cette adéquation que nous établissons entre la Montagne et le Jéhovah biblique.

Que l'Homme-au-crayon-magique, dit-il, prenne garde à la montagne. Elle n'aimait peut-être pas sortir de son mystère et du silence: elle lui en voudrait peut-être de faire son image, surtout s'il ne la réussissait pas bien ${ }^{15}$.

Cette manière de concevoir les rapports de l'artiste avec l'objet qu'il peint n'est pas exclusive à Orok, le primitif qui sculpte au couteau ses figurines dans la saponite; Pierre entretient des craintes semblables à celles d'Orok lorsqu'il doit peindre la ville de Paris sur l'ordre de son maître, Augustin Meyrand.

Il lui semblait que la noble ville allait souffrir comme un affront qu'il osât, après tant d'autres, tâcher de fixer quelque aspect de son visage. Elle ne le souffrirait pas, pensait-il, elle se défendrait contre lui bien autrement que l'Ungava ${ }^{16}$.

La crainte superstitieuse d'offenser l'être qu'on peint - soit en le peignant, puisqu'on capte son âme, soit en le peignant mal et en se rendant coupable par le fait même d'un acte doublement sacrilège - la crainte superstitieuse donc n'est pas étrangère au sentiment d'anxiété ressenti par le héros au moment où il s'apprête à peindre pour la première fois la Montagne. La tâche à entreprendre lui semble une lutte ardue qu'il doit mener à bonne fin. Le passage qui suit fait état des liens subtils mais réels qui sous-tendent l'adéquation Montagne-Jéhovah et rapprochent l'acte de 
création que le héros s'apprête à accomplir d'une lutte avec la présence divine comme il en est question dans le récit de la nuit au terme de laquelle Jacob reçut la confirmation de sa mission divine ${ }^{17}$.

Au matin, vite debout, son premier regard fut pour la Solitaire. Mais plutôt la nomma-t-il en son cceur: la Resplendissante. Encore qu'à cette heure, faisant écran au soleil, elle fût plutôt menaçante. Indubitablement, c'était au soleil déclinant, à l'heure même où elle lui était apparue, qu'il fallait la saisir.

Mais quel mot étrange! Juste cependant, car c'était de cela qu'il s'agissait, cela qu'il voulait entreprendre: une sorte de corps à corps avec cette présence implacable. La saisir donc! II en avait peur maintenant. $\hat{A}$ sa joie profonde se mêlait de la timidité. L'appréhension de faire affront à la montagne, ou à soi-même ${ }^{18}$.

Les figures des patriarches Moïse et Jacob, les motifs du Buisson ardent et de la Lutte avec une présence divine ont pour rôle précis d'incarner dans le roman ce caractère que le code culturel par son mythe de l'artiste lui confère, savoir celui d'un être d'exception inspiré par la divinité, “cet enfant rare entre les hommes, celui qui ouvre leurs yeux, celui qui ouvre aussi entre eux de grandes portes soudaines de communications ${ }^{19}$ ". Sous ce rapport, le mythe de l'artiste tel que la société le conçoit et qui s'exprime par la littérature ne fait que rendre perceptible au niveau figuratif certains traits marquants du pattern archétype de l'Enfant divin ${ }^{20}$.

La deuxième phase obligée du processus de la quête, l'accomplissement de cette quête, se range sous l'égide de l'archétype du Voyageur au monde souterrain. Plusieurs héros de la mythologie gréco-romaine én particulier ont incarné de diverses manières les faisceaux de caractéristiques propres à cet archétype. Trois mythes qui participent les uns de la culture populaire, les autres, de la culture savante, nous semblent structurer le roman de Gabrielle Roy et reconstituer, Iorsque nous en coordonnons leurs divers éléments, le pattern archétype fragmenté des exploits du Voyageur au monde souterrain. Il s'agit des mythes de l'artiste, du coureur de bois, et de la montée vers le Nord, qui se manifestent ${ }^{21}$ dans des structures de type culturel d'une part et stylistique d'autre part, puisque l'auteur qui les emprunte à sa société les transforme en principes organisateurs de son œuvre. Pour désigner le premier type de structures, nous emploierons l'expression "code culturel", pour le second, "code de l'œuvre". II nous faut également préciser que nous utiliserons le mot "mythe" dans le sens particulier que lui confère Roland Barthes dans Mythologies 22: les mythes de la vie quotidienne sont une "parole", un mode de communication, un système qui permet aux locuteurs d'appréhender des réalités souvent considérées comme banales, sous leurs aspect de signifiants valorisés pour une collectivité donnée.

Beaucoup plus important dans l'économie du roman que les quelques mythes classiques judéo-chrétiens et gréco-romains auxquels certains motifs mythologiques secondaires de l'écrivain font brièvement allusion, ces trois mythes toujours vivants de la société québécoise fournissent à Gabrielle Roy son lot de patterns archétypes, le canevas de son intrigue, les 
TABLEAU I

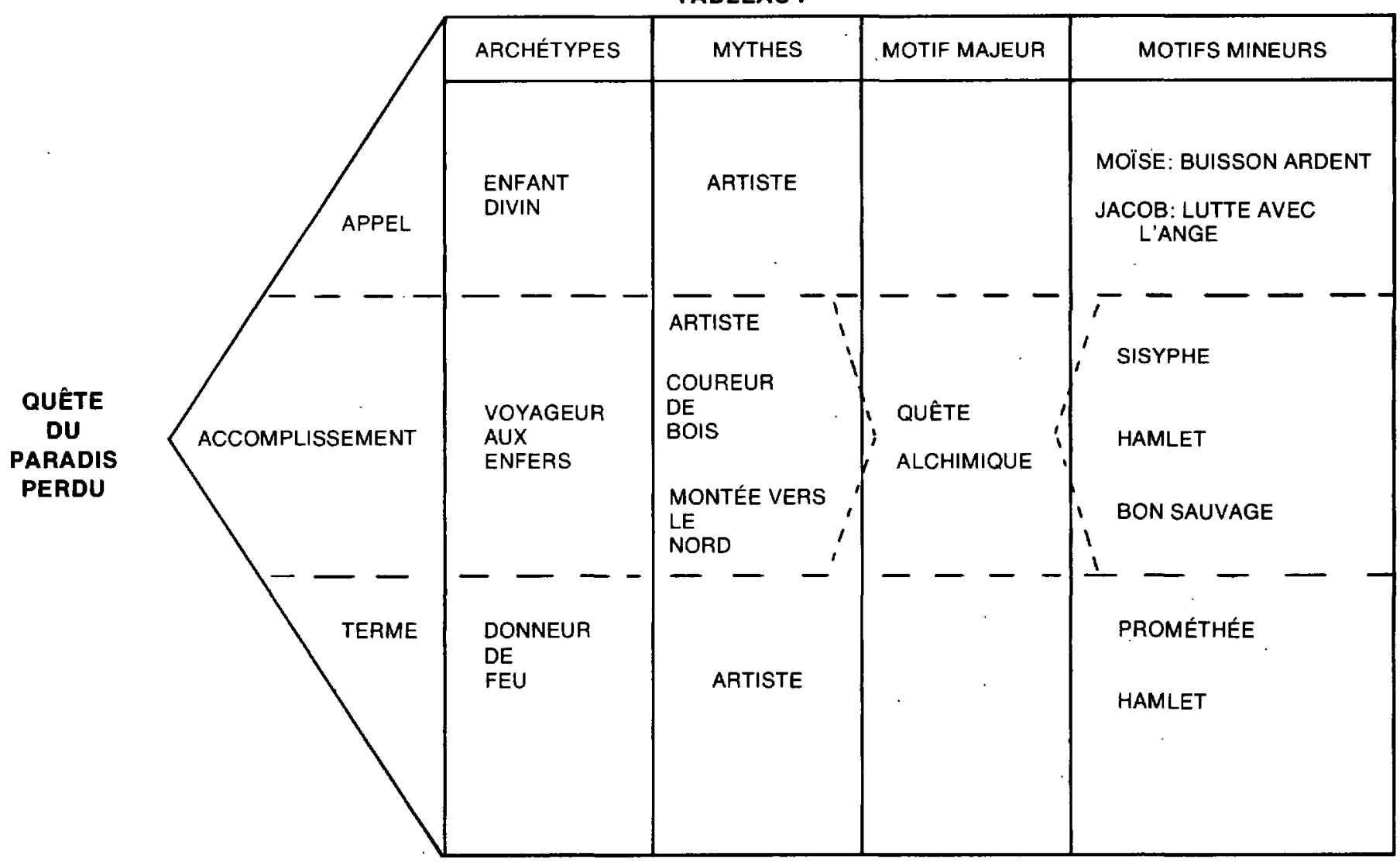


TABLEAU II

Niveaux de figuration *

\begin{tabular}{|c|c|c|c|c|c|c|c|c|c|c|}
\hline $\begin{array}{l}\text { NIVEAUX } \\
\text { D'ANALYSE }\end{array}$ & $\begin{array}{c}\text { TYPES } \\
\text { DE STRUCTURES }\end{array}$ & $\begin{array}{l}\text { NIVEAUX } \\
\text { DE FIGURATION }\end{array}$ & $\begin{array}{c}\text { TYPES } \\
\text { DE SYSTĖMES }\end{array}$ & & & & $\begin{array}{l}\text { YPE } \\
\text { YNIT }\end{array}$ & & & \\
\hline $\begin{array}{l}\text { HORS- } \\
\text { MANIFESTATION }\end{array}$ & LOGIQUES & CONCEPTUEL & ARCHÉTYPES & \multicolumn{7}{|c|}{ LE PARRICIDE } \\
\hline \multirow{2}{*}{ MANIFESTATION } & $\begin{array}{l}\text { SÉMANTIQUES } \\
\text { ET } \\
\text { SÉMIOLOGIQUES }\end{array}$ & FIGURATIF & MYTHES & \multicolumn{4}{|c|}{ L'CEDIPODIE } & \multicolumn{3}{|c|}{ L'ORESTIE } \\
\hline & $\begin{array}{l}\text { STYLISTIQUES } \\
\text { ET } \\
\text { CULTURELLES } \\
\text { (code culturel } \\
\text { et code de } \\
\text { l'cuure) }\end{array}$ & ANTHROPOMORPHE & $\begin{array}{l}\text { LITTÉRATURE } \\
\text { MYTHOLOGIQUE } \\
\text { (romans, contes, } \\
\text { théâtre, etc.) }\end{array}$ & ب & 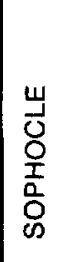 & $\frac{\text { 亗 }}{\frac{\bar{a}}{\bar{a}}}$ & 㟧 & 崖 & 导 & 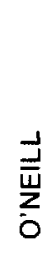 \\
\hline
\end{tabular}

* Voir Philippe Hamon, “Pour un statut sémiologique du personnage ”, Littérature, n 6, mai 1972, p. 105. 
grands traits de la psychologie de ses personnages, ses figures de style, bref, si notre hypothèse se vérifie, la structure même de son roman. Tous les motifs mythologiques secondaires de l'œuvre ne vont-ils pas s'intégrer et trouver leur unité dans ces trois mythes fondamentaux qui, à leur tour, se fondent dans un certain mythe de la quête, que nous tenterons d'identifier plus loin mais que nous pourrions d'ores et déjà considérer comme la signification dernière de tout le roman?

Cependant établissons d'abord les rapports qu'entretiennent entre eux, au sein même de l'œuvre, ces trois mythes de notre culture. Stanislas Lanski, l'ami-peintre parisien de Pierre Cadorai, connaît, quelques moments avant la mort du héros, une expérience révélatrice que le narrateur nous livre ainsi: "Ce soir, d'une manière inexplicable, Stanislas s'était senti grandir dans l'âme - ce qui, le métier appris, est peut-être la seule manière de grandir en art. II croyait apercevoir l'amorce d'une longue route solitaire, s'y trouvait comme déjà poussé ${ }^{23}$. "Au soir de la mort de Pierre, Stanislas qui s'était décrit auparavant comme l'un des "trois ou quatre mille petits peinturlureux de Paris ${ }^{24}$, , entrevoit ce qu'est une vraie vie d'artiste. Lui qui auparavant reprochait à Pierre de vivre en solitaire, il comprend enfin que l'on ne devient véritablement un créateur qu'au terme d'un long cheminement solitaire, d'une longue quête. II a fallu qu'il s'élève en luimême pour saisir cette vérité. Un processus de purification est amorcé chez Stanislas, c'est le sens du segment: "s'y trouvait comme déjà poussé". Pour parvenir au terme de sa propre quête, il lui faudra renoncer à l'alchimie trompeuse de la bohème, à "ce feu étrange des inlassables parlottes, de principes constamment brassés ${ }^{25}$ ".

L'histoire de Pierre Cadorai, c'est l'histoire de cette longue quête. C'est le récit, étape par étape, de cette démarche de purification mise au service de l'art. Résumant au bénéfice de Stanislas les longues années de nomadisme qui ont précédé sa venue à Paris, le héros pouvait dire:

II me faudrait n'avoir que vingt-cinq ans, [...]. Mais à cet âge, avec mon associé, un nommé Sigurdsen, alias le grand Steve, je piégeais les bords du lac Caribou, et entre moi et les Beaux-Arts s'étendait une distance infinie: les Territoires du Nord-Ouest, les savanes du Nord manitobain, l'Ungava; de plus la route à faire en moi-même ${ }^{26}$.

Ce passage joint les notions de distance physique et de distance morale; l'errance du coureur de bois sous-tend la démarche de l'artiste. Le mythe de la montée vers le Nord - en littérature québécoise, cet attrait nordique manifeste toujours une préférence accordée aux valeurs spirituelles d'idéal, de courage, de sacrifices; de dépassement de soi, que l'on se souvienne des héros de Félix-Antoine Savard, de Léo-Paul Desrosiers pour n'en nommer que quelques-uns - le mythe du Nord viril et purificateur inspire donc la poussée aventureuse de Pierre qui tente d'atteindre le delta du Mackenzie et que nous suivons jusqu'à une bourgade au nom symbolique de FortRenonciation. Pierre y fera le choix capital qui aiguillonnera de façon définitive son existence; les hantises de l'inconnu, "cette faim des endroits perdus ${ }^{27}$ "lui fera renoncer à Nina, à l'amour. Sans encore savoir pourquoi, il opte pour la solitude. Les portages lui ont peut-être déjà 
appris que pour voyager loin il faut renoncer à tout ce qui n'est pas le strict mınimum devant assurer la vie.

Tant est grande et permanente l'influence de la vie de coureur de bois sur le héros, elle lui fournit plus tard à Paris les points de référence qui lui permettent de concevoir sa tâche de peintre. Le décrivant au travail dans son atelier, le narrateur dira:

Sa journée faite, il ressemblaıt bien plus à quelqu'un ayant escaladé une montagne qu'à un homme dont le but à atteindre est en son âme. Lui-même avait du reste l'impression de s'être tenu toute la journée sur le surplomb étroit d'un rocher, ou encore d'arriver au terme d'un portage extrêmement pénible. D'où venait que, du simple effort de son esprit, il ressentait plus de fatigue que, naguère, d'avoir porté sur son dos une charge de cent livres, ou d'avoir pagayé à travers de turbulents remous? II lui arrivait en déposant ses pinceaux de chercher autour de lui d'un air égaré où il se pouvait bien trouver. Auprès du plus difficile entre tous les fleuves, ce Churchill plein de rapides? Ou dans l'infinie Saskatchewan? L'illusion du portage était si forte qu'il retrouvait le geste d'autrefois pour dégager ses épaules d'un sac pesant ${ }^{28}$.

De toute évidence, les données des mythes du Nord et du coureur de bois s'insèrent dans le motif mythologique de la quête entreprise par l'artiste. Habilement semés au long du récit, plusieurs indices font germer dans l'esprit du lecteur l'idée que la quête du héros est une descente aux enfers. Autre appellation figée qui désigne un ensemble paradigmatique de mythes, ce qu'il est convenu de désigner par ce terme est une illustration, au niveau figuratif, du pattern archétype du Voyageur au monde souterrain. La descente aux enfers est le fait de héros qui n'ont souvent rien d'autre en commun, qui obéissent à des mobiles différents. Quant aux modalités de l'exploit, elles varient d'un mythe à l'autre. Cependant, des constantes se dégagent des différents récits mythologiques. Toujours on descend aux enfers pour rechercher un certain bien. La descente est donc une quête. C'est aussi une entreprise périlleuse. Et le voyage au monde des profondeurs entraîne toujours une transformation de qui l'a effectué parce qu'on en rapporte une certaine science - quel que soit d'ailleurs le but poursuivi en entreprenant le voyage - une sagesse précieuse pour le temps qui reste à vivre.

La suite de l'analyse nous permettra de relever ces segments anaphoriques qui créent le motif mythologique de la quête-descente aux enfers. Vers la fin du roman, Pierre qui travaille fébrilement dans son atelier parisien, coordonne en une même vision ses efforts pour peindre sa Montagne et un paysage imaginaire, véritable scène d'un monde des profondeurs qui en se précisant devient le souvenir des moments précédant sa découverte de la Montagne dans l'Ungava.

II approchait de son but - l'ignorant encore, mais assuré qu'en le voyant il le reconnaîtrait. II éprouvait la sensation d'une lumière venant à soi et, parfois, ce sentiment de contourner une masse sombre avant d'entrer dans le plein jour.

[...]

Comme autrefois, dans l'Ungava, avant que ne lui apparût la monta- 
gne étincelante, l'impression lui venait de n'avoir plus que quelques pas à faire pour être ébloui de clarté. L'impression était si forte que, reprenant son pinceau, il lui semblait plutôt soulever son canot, tandis qu'en bas l'eau dangereuse l'attirait ${ }^{29}$.

Ce souvenir nous ramène loin en arrière dans le roman, plus précisément au début de la deuxième partie, alors que Pierre arrive par la rivière des Mille-Tonnerre près de l'endroit où il découvrira la Montagne. Dans le récit, ce qui précède immédiatement, c'est-à-dire la première partie, nous fait suivre le héros qui s'engage seul sur une rivière inconnue puisqu'elle ne figure «Sur aucune des cartes qu'il avait pu avoir des Terres et Forêts, ni non plus en ses rencontres récentes en avait-il entendu parlé 30 ". Cette rivière sans nom, "ensorcelante", dont l'eau est "presque noire", coule rapidement entre de hautes parois rocheuses couvertes de lichens éclatants qui aux yeux de Pierre transforment ces falaises en une "fresque continue", et la rivière elle-même "en tapisseries anciennes à l'effroyable réseau sur fond sombre, au reste presque tout couvert par le motif, de lianes, de fleurs et de brillants petits points d'or ${ }^{31}$ ". Le couloir sombre de la rivière dont les eaux rapides happent Pierre qui ne pourra rebrousser chemin et.fera par la suite naufrage, ces eaux miroitantes deviennent tapisseries aux motifs évoquant les forêts impénétrables des légendes médiévales. Traitant de la forêt hantée dans l'Art fantastique, Marcel Brion soutient que:

La peur de l'homme moderne lorsqu'il marche, seul, dans la forêt vierge, l'inquiétude de l'homme préhistorique lorsqu'il se voyait entouré de forêts millénaires où les arbres morts de vieillesse et les arbres vivants formaient un inextricable chaos, survivent dans cette Identification de la forêt d'une part, de la mort et de l'enfer de l'autre, qui se perpétue même dans les époques de civilisation ${ }^{32}$.

Dans Mythes et Symboles, Mircéa Eliade abonde dans le même sens. Il écrit: "ll y a là déjà un symbole de la mort: la forêt, la jungle, les ténèbres, symbolisent l'au-delà, les Enfers ${ }^{33}$ ". Cette rivière dangereuse nous apparaît comme le Styx de la mythologie grecque, l'entrée au monde souterrain. Si à la suite de Brion, nous admettons que "la forêt est l'endroit où l'on se perd, matériellement, moralement ${ }^{34}$ ", il ne faut pas oublier cependant que le héros véritable surmonte cette épreuve de la perte en forêt et qu'il ne parvient au salut - le château, archétype antagoniste de ce couple inséparable, forêt/château - que parce qu'il a encouru le risque de se perdre. Voyant les rapides de la rivière, Pierre pense: “le désastre certain... mais, peut-être, malgré tout, le salut... ${ }^{35}$ ". Tel est à notre avis le sens des motifs mythologiques de la descente aux enfers, le salut par la perte.

La description que nous fait le narrateur du pays où aborde Pierre à la suite de son naufrage ne laisse subsister aucun doute quant à ce qu'évoque le paysage désolé qui s'étale devant le héros.

Devant lui, grise, monotone, s'étendait sans fin une stérıle forêt de petites épinettes ınanımées. Aussi loin qu'il pouvait voir, II n'aperçut rien d'humain, ni fumée, ni habitation, ni même de piste en cet enchevêtrement comme laineux des arbres rabougris. Même au Bas- 
Mackenzie il n'avait jamais surpris aspect du monde plus redoutable et triste. If lui apparut que n'avaient peut-être jamais été que des ennemis, appliqués à le railler, ces appels d'âme qu'il avait tant de fois reçus ${ }^{36}$.

La première partıe du roman s'achève donc avec l'arrivée du héros aux enfers.

La conjonction rivière-forêt que nous trouvons dans l'œuvre s'avère des plus fécondes pour la compréhension du motif de la descente aux enfers. A propos de la forêt Marcel Brion nous fournit une autre information susceptible d'expliquer le pourquoi d'une telle conjonction :

La forêt est un monde inhumain, d'où l'homme est exclu, où il n'est admis qu'à regret. Les êtres et les choses, les plantes et les animaux y confondent leurs formes, leurs couleurs, leurs propriétés. C'est un monde irrationnel, où se rassemblent, féroces et tranquilles les éléments hostiles, un monde autre, qui ne devient accueillant que dans les clairières aux endroits où la cognée du bûcheron a ouvert un passage pour la lumière ${ }^{37}$.

Deux ıdées sont à retenir de cette citation, la première, que le monde de la forêt est informel de par sa confusion des formes, des couleurs, des propriétés, la seconde, qu'il s'agit d'un monde "autre" que celui où l'homme a coutume d'évoluer. La rivière sur laquelle Pıerre s'est lancé a bientôt un cours si rapide, des «eaux si agitées qu'elles brouillaient, effaçaient les images, et il devait se contenter au passage de noter les teintes et les effets $^{38}$ ". L'enchevêtrement incroyable "de lianes, de fleurs et de brillants petits points d'or ${ }^{39}$ " se dissout en un magma de couleurs. La descente aux enfers, nous pensons devoir l'affirmer à ce stade de notre étude, préfigure une plongée dans l'irrationnel, ce monde «autre" par excellence quı n'est pas soumis à la lumière de la pensée raisonnante.

Le code culturel nous fournit un exemple historique d'un type de quête qui se voulut plongée aux racınes de l'être, la quête alchimique. C. J. Jung, le psychologue de l'inconscient, s'est intéressé à cette ascèse que constitue l'alchimie véritable, non point celle qui se donnait pour but la transmutation des matériaux vils en or mais celle qui se consacrait à la recherche de la pıerre philosophale, l'or spırituel, et lui a consacré au moins un essai, Psychology and Alchemy ${ }^{40}$. Reprenant, oserons-nous écrire, à son Insu, l'intuition heureuse du conteur et poète E. T. A. Hoffmann qui dans le Vase d'or assoclait la reconquête de l'unité intérieure et l'acquisition d'une vraie sagesse aux étapes d'une purification du héros inspirée du grand œuvre alchimique, Jung compare ce processus alchimique au développement normal de la personnalité qui doit se faire dans le maintien d'un équilibre toujours précaire entre l'ego et les forces de l'Inconscient. L'alchimie se proposait d'atteindre la pierre philosophale par une fusion harmonieuse des éléments contraires de la nature, la terre, l'air, le feu et l'eau. Manifestant en cela son étrolte parenté avec la pensée mythique qui «procède de la prise de conscience de certaines oppositions et tend à leur médiatıon progressive ${ }^{41}$, elle établissait une identification rigoureuse entre les processus de transformation de la materia prima dans le creuset et 
de transmutation de l'être de l'alchimiste dans l'ascèse accompagnant l'accomplissement du grand œuvre. Au terme de ces deux processus concomitants l'alchimiste parvenaıt à l'iliumination. L'illumination, c'est l'expérience extatique de l'intégration avec tout ce qui est, c'est la fusion harmonieuse du moi individuel intégré dans le grand tout de la nature.

Expérience mystique tant religieuse qu'artistique, elle constituerait selon Jung le terme idéal du développement de la personnalité. Rares sont ceux qui y parviennent il taut en convenir. Le héros de Gabrielle Roy cependant appartient au petit nombre des élus. Au maître peintre Augustin Meyrand qui lui pose cette question: "C'est de peindre qui vous importe ${ }^{42}$ ?", le héros répond par l'affirmative. Suit alors un moment de réflexion où il prend conscience que:

le froıd, la faim, ces longs voyages de solitaire, la misère de vivre, tout cela il s'aperçut l'avoir accepté, pouvoir accepté pire, à condition que tout fût dépassé, transcendé, c'est-à-dire relié de quelque manière à tout ce qui est ${ }^{43}$.

II n'y a donc rien d'étonnant au fait que lorsqu'il peint son autoportrait, Pierre se représente avec les attributs de deux de ses préfigurations dans le récit, le caribou et le peuplier-tremble. Stanislas qui contemple le chefd'œuvre peint par son ami quelque temps avant de mourir y voit:

de curieuses protubérances, une suggestion de bois de cerf peutêtre, que prolongeait comme un mouvement de feuillages ou d'ombres. Cependant, la pupille, quoique dilatée, était bien celle d'un homme, d'une lucidite, d'une tristesse intolérables. [...] Qu'avait donc voulu suggérer Pierre? Quelle alliance étroite de l'âme avec les forces primitives? Ou la haute plainte d'une créature en qui se fût fondue l'angoisse de tuer et d'être tuée? Le portrait attirait comme vers une insolite région de la connaissance dont les arbres, avec leurs sombres entrelacements, donnaient quelque idée. Son attrait était dans cette sorte de fascination qu'il exerçait au rebours de la clarté, vers les torturantes énigmes de l'être ${ }^{44}$.

II semble donc que le héros ait atteint en sa personne et en sa peinture à cette fusion du végétal, de l'animal et de l'humain. Mieux encore, quand Stanislas reconnaît dans le tableau l'empreinte du génie et qu'il déclare à Pierre: "Eh bien, cette fois, mon vieux, sur le mur ta main laisse son empreinte 45 ", l'allusion biblique à la main qui traçait des caractères mystérieux sur le mur du palais de Babylone manifeste que par son art Pierre en est arrivé à exprimer le divin. En langage alchimique, Pierre parvient à la dernière étape du grand œuvre, l'illumination. Tout comme les alchimistes d'autrefois, il savait maintenant qu'une secrète unité se cachait sous les apparences complexes de la nature. II ne lui reste plus, comme l'adepte de l'alchimie qu'à attendre que la lumière vienne à lui. Cette expérience extatique qui couronnait la vie des alchimistes authentiques a son pendant dans l'œuvre de Gabrielle Roy dans la scène finale de la vision de la Montagne qui vient vers le héros mourant. Le terme d'illumination lui-même se retrouve dans le texte pour désigner ce moment où Pierre saisit "le complexe écheveau de coloris, d'ombre et de clarté ${ }^{46}$ " qu'il se hâte de jeter sur sa toile dans un dernier sursaut d'énergie. 
Les motifs mythologiques propres à une mythologie spécifiquement québécoise, ceux qui relèvent de l'errance du coureur de bois et de la montée vers le Nord, s'intègrent donc harmonieusement à ce motif plus vaste car il enserre tout le récit, inspiré par cette préfiguration historique de la quête alchimique devenue, dans l'œuvre même, l'équivalent de l'ascèse de l'artiste. Quelques motifs mineurs développent encore ces motifs plus généraux auxquels ils se rattachent. La préfiguration littéraire de Hamlet, par exemple, peut être reliée en partie au motif de la quête alchimique. Le prince du Danemark, tout comme Pierre qui se reconnaît en lui, plonge au plus profond de lui-même à la recherche de répónses aux "torturantes énigmes de l'être". C'est le sens du célèbre monologue "to be or not to be " que cite partiellement le narrateur.

Une autre préfiguration littéraire, le Bon Sauvage, viènt se greffer au motif mythologique de l'errance du coureur de bois. Écrasé par les chefs-d'œuvre qu'il a contemplés au Louvre, ébloui par les grandeurs d'un Paris chargé de gloire, Pierre subit le choc de l'aliénation. Dépouillé de son identité, après un passage dont le ton évoque les romans du dixhuitième siècle, il en vient à s'interpeller de la façon suivante: "Pauvre Sauvage, retourne là d'où tu viens, là où seulement tu peux être quelqu'un, quelque chose ${ }^{47}$." Ce motif du Bon Sauvage évoluera d'une façon intéressante au cours des pages qui vont suivre. Stanislas Lanski adhérera à ce mythe qui pour lui ne gardera que ses connotations de pureté, d'authenticité. Ainsi, après la rencontre avec Pierre, alors que les deux campent sur le bord de la Seine, Stanislas jette sur les êtres un regard neuf. "Allongé face au ciel, Stanislas observa qu'il les [étoiles] remarquait bien pour la première fois depuis longtemps ${ }^{48}$." Pierre et lui communient d'ailleurs au même sentiment de pureté originelle qui les fait se sentir «nulle part et partout à la fois sur la terre des hommes ${ }^{59}$ ". Cette adhésion naïve de Stanislas au mythe dans lequel la figure de son ami s'insère d'emblée, ne contribue pas pour peu à son évolution morale dont il a déjà été fait mention.

Ainsi sommes-nous en mesure de constater que les préfigurations littéraires, loin d'être de simples ornements du récit; constituent de véritables principes générateurs de ce même récit. A titre d'exemple, nous citerons la préfiguration shakespearienne dont le narrateur tire un double parti. Si le personnage de Hamlet dont le texte du monologue, même tronqué, nous livre encore les angoisses qu'il nourrit sur la condition humaine, préfigure le héros de la Montagne secrète dans sa quête d'unité intérieure, un autre passage de la même tragédie fait le pont entre la quête de l'artiste et sa mission en regard de l'humanité. If s'agit des vers suivants qui font ressortir le pathétique de toute vie humaine, livrée au bruit et à la fureur, en proie aux hésitations, aux tergiversations comme aux sursauts d'énergie, vie qui n'aspire en fin de compte qu'à trouver son sens dans le récit qu'on en fera.

If thou didst ever hold me in thy heart, I

Absent thee from felicity awhile, I

And in this harsh world draw thy breath in pain.!

To tell my story ${ }^{50}$. 
Ce que Hamlet mourant attend de son ami Horatio, c'est ce que tout homme attend de l'artiste, écrivain, peintre, sculpteur ou autre, qu'il arrache sa propre vie si brève au mouvement perpétuel de l'immanence, au cycle éternel de tout ce qui naît, vit, meurt, se décompose et renaît sous une autre forme, qu'il l'arrache à l'angoisse de ce mouvement, de cette fuite incessante du temps, en lui donnant par la magie de son art accès à une forme de transcendance. Cette idée probablement aussi vieille que la civilisation occidentale, et dont André Malraux s'est fait le défenseur sans doute le plus éloquent à notre époque, en particulier dans la Métamorphose des dieux et les Voix du silence, cette idée donc relève de notre code culturel, appartient à notre façon de concevoir l'artiste.

Au cours du récit différents personnages expriment des opinions concernant l'artiste, son rôle, ses rapports avec les hommes, dont la somme constitue une réplique assez fidèle, assez complète de la conception qu'on se fait de l'artiste dans notre société, de ce que nous sommes convenus d'appeler notre "mythe de l'artiste".

Les lignes de force de ce mythe de l'artiste tel que nous pouvons le lire tout au long du roman de Gabrielle Roy, la Montagne secrète, sont au nombre de trois: la médiation, la communication, la communion, toutes trois réductibles à ce dénominateur commun qu'est la mission prométhéenne de l'artiste. Car c'est bien de cela qu'il s'agit. La société confère à l'artiste les caractéristiques et le rôle de la figure-archétype du Donneur de feu qui pour notre civilisation occidentale s'est incarné dans le Prométhée du mythe grec.

La troisième phase obligée du processus de la quête, le terme de la quête, s'inscrit donc sous l'égide du Donneur de feu. L'ascèse de l'artiste, cette purification qui est une conquête de lui-même, il ne la mène à son terme que parce qu'il lui faut se rendre digne de sa mission.

Le premier aspect de sa mission, la médiation, apparaît assez tôt dans le récit lorsque, par exemple,le narrateur mentionne à propos du vieux Sigurdsen "que les images les plus tenaces de sa vie ne lui venaient pas de son propre regard, mais de ce qu'on les lui avait racontées, de ce qu'on les avait dépeintes à ses yeux ${ }^{51}$ ". Déjà dans la dédicace le romancier soulignait cette importante fonction de l'artiste en reconnaissant devoir sa connaissance du Grand Nord à René Richard, peintre et auteur de récits. En tant que partie du système préfiguratif, la dédicace doit être reliée à ce caractère prométhéen du rôle de l'artiste qui crée un monde parallèle, à la fois autre et semblable à la réalité, mais qui parle infiniment mieux à l'esprit des hommes parce qu'un homme l'a, "à son propre feu intérieur, coulé, fondu, pour ensuite le mouler à son gré en une matière qui [n'est] désormais plus qu'humaine, infiniment poignante ${ }^{52}$ ".

La médiation suppose la communication. Si un objet du monde réel ne se met à exister qu'au moment où il a été capté dans un regard humain ${ }^{53}$, si l'art transmute un objet en lui conférant une "sur-vie", l'œuvre de l'artiste ne vit vraiment qu'au moment où elle devient un moyen de com- 
munication. Après avoir réussi ses premières pochades de la Montagne, Pierre se découvre le désir de faire connaître son œuvre aux hommes.

II s'aperçut qu'il pensait à des hommes, des inconnus, une multitude. II revait d'eux, d'une entente entre eux et lui, _ d'une entente avec des inconnus, - lui qui, toute sa vie, jusqu'ici, s'était sans cesse éloigné des hommes.

\section{Éloigné? Ou rapproché?}

Tout à coup, l'inonda le sentiment d'avoir pour eux seuls osé ce qu'il avait fait. Et pour qui d'autre l'eût-il tenté ${ }^{54}$ ?

Ce sont là des accents prométhéens. Prométhéen encore son désir d'accéder à la liberté par l'art, de libérer les hommes qui communiquent avec lui et entre eux grâce à son œuvre.

II pensait à cette impression qu'il avait maintes fois éprouvée d'avoir en la poitrine un immense oiseau captif _ d'être lui-même cet oiseau prisonnier - et, parfois, alors qu'il peignait la lumière ou l'eau ou quelque image de liberté, le captif en lui, pour quelques instants s'évadait, volait un peu de ses ailes. Songeur, à demi étendu sur la mousse, Pierre entrevoyait que tout homme avait sans doute en sa poitrine pareil oiseau retenu qui le faisait souffrir. Mais, lorsque lui-même se libérait, pensait Pierre, est-ce que du même coup il ne libérait pas aussi d'autres hommes, leur pensée enchaînée, leur esprit souffrant 55 ?

L'immense oiseau dont il est question à deux reprises dans la citation évoque, bien sûr, l'aigle du récit mythologique. Cet aigle, instrument du châtiment qu'un Zeus jaloux de ses prérogatives exerce contre la personne du Titan enchaîné, quelle est sa signification ultime dans le récit mythologique? On est souvent châtié par où l'on a péché. Dans le cas du mythe qui nous occupe il semble que l'instrument du supplice entretienne quelque secret rapport avec la nature du crime commis. Dans son étude sur les Structures anthropologiques de l'imaginaire, Gilbert Durand soutient que:

L'outil ascensionnel par excellence, c'est bien l'aile dont l'échelle du chamane ou l'escalier de la ziqqurat n'est qu'un grossier succédané. Cette extrapolation naturelle de la verticalisation posturale est la raison profonde qui motive la facilité avec laquelle la rêverie volante, techniquement absurde, est acceptée et privilégiée par le désir d'angélisme. [...] Bachelard, après les chamanes mystiques l'a vu très profondément, l'aide est déjà moyen symbolique de purification rationnelle. D'où il résulte paradoxalement que l'oiseau n'est presque jamais envisagé comme un animal, mais comme un simple accessoire de l'aile. [...] L'oiseau est désanimalisé au profit de la fonction. Une fois de plus ce n'est pas au substantif que nous renvoie un symbole, mais au verbe. L'aile est l'attribut de voler, non de l'oiseau. Les images ornithologiques renvoient toutes au désir dynamique d'élévation, de sublimation 56 .

Si, à la suite de Durand, nous tenons le feu dont il est question dans le mythe de Prométhée pour "un simple succédané symbolique de la lumièreesprit ${ }^{57}$ ", l'exploit de Prométhée se présente alors comme un rapt de l'immortalité commis en faveur des hommes. L'aigle, symbole d'une volonté de transcendance, manifeste dans le châtiment ce pourquoi le héros est puni. 
L'ambition de Pierre de “donner la parole aux bêtes comme aux hommes, à tout ce qui connaissait la souffrance de vivre ${ }^{58}$ "s'inscrit dans sa mission prométhéenne qui est de faire survivre ce qui est voué à la mort. Quand Orok constate que «l'Homme-au-crayon-magique qui semblait travailler à élucider le mystère de la vie, de plus en plus aux autres le découvrait ${ }^{59}$ ", nous pouvons en conclure que Pierre assume maintenant sa deuxième fonction en tant qu'artiste, la communication.

La communion devient possible, elle, quand l'œuvre porte l'empreinte du génie. S'il a encore un long apprentissage à faire en ce qui regarde la peinture, Pierre est en mesure dès son arrivée à l'Académie Meyrand de présenter au regard du maître des dessins d'une rare qualité. Ce dernier s'en trouve

comme ravi en songe. Ici rien n'arrêtait l'élan créateur, ne le desservait ni ne le trahissait. Idée, forme, matière, tout cela n'était qu'un: la vision même d'une âme, et si claire, si limpide, qu'on y pouvait entrer sans heurt comme dans la vérité 60 .

L'autoportrait peint par le héros peu avant sa mort et dont nous avons déjà parlé, crée chez qui le regarde un semblable sentiment de communion à la totalité de l'univers.

La figure de Prométhée se profile derrière les données du code culturel cristallisées dans le mythe de l'artiste mais quelques brèves allusions et évocations ne constituent toujours qu'un motif mineur. Le motif mineur des labeurs incessants de Sisyphe, manifesté lorsque Pierre s'astreignait sous la gouverne d'Augustin Meyrand à des exercices picturaux "si pénibles, si absurdes qu'il y perdait toute liberté et presque son identité ${ }^{61}$, cette tâche dépourvue en l'occurrence de tout caractère punitif, s'inscrit tout naturellement dans une série de cinq épreuves dont le héros doit triompher et qui font partie du processus de sa purification. C'est donc dire que le motif mythologique de Sisyphe, tout comme celui de Prométhée, comme les préfigurations littéraires du Bon Sauvage et de Hamlet, se fondent dans le grand pattern unificateur de la quête alchimique, exemplaire de l'évolution et de l'élévation de l'homme et de l'artiste.

Dans l'ensemble, les préfigurations littéraires, du moins celles qui ont été énumérées et dont le fonctionnement a été analysé par White sont relativement peu nombreuses bien qu'elles soient importantes pour la structure de l'cuure. Une des raisons susceptibles d'expliquer cette relative atonie du système préfiguratif traditionnel pourrait tenir dans le fait que l'œuvre crée sa propre "mythologie" en quelque sorte, s'organise selon un système préfiguratif qu'elle engendre elle-même pour une bonne part.

Ce système préfiguratif propre à l'œuvre repose sur les figures de style, métaphores et comparaisons par lesquelles le héros, grâce à la conscience qu'il acquiert progressivement de ce qui l'unit à ses préfigurations, peuplier-tremble et caribou en particulier, évolue vers une complète connaissance et possession de lui-même.

Ces métaphores et comparaisons, groupées en séries homogènes et hétérogènes qui se développent en mọtifs, créent des liens entre les parties 
du récit, donc exercent pleinement leur fonction anaphorique et constituent ainsi les fondements du système préfiguratif analogique qui régit toute l'œuvre. A partir de rapports de similitude et d'identification qui s'élaborent au fur et à mesure entre certains êtres à caractère anthropomorphe et le héros - que nous qualifierons de préfigurations explicites parce qu'elles finissent par s'inscrire dans le champ de conscience du héros - nous pouvons inférer l'existence d'au moins deux autres types de préfigurations implicites qui se situent au niveau des rapports métaphoriques qu'entretiennent les personnages humains et le héros, et ces mêmes personnages entre eux mais toujours en relation avec la figure centrale du héros. Ces rapports métaphoriques sont si importants qu'ils gouvernent l'apparition des personnages dans le roman. Chaque personnage introduit dans le récit s'insère immédiatement dans un système fondé sur des rapports analogiques. Gédéon, le chercheur d'or matériel, représentant de l'humanité entière en ce qu'il est un visage de "l'espérance infinie, l'infinie attente des hommes ${ }^{62}$ " correspond à Pierre, le chercheur d'or spirituel.

À titre d'exemple pour le second groupe de préfigurations implicites, nous citerons ce motif qui regroupe Steve Sigurdsen, que Pierre considère comme "le frère robuste de son âme peut-être trop frêle ${ }^{63}$ ", le Père Le Bonniec, et le maître Augustin Meyrand, tous trois protecteurs de cet «enfant rare "qu'est l'artiste.

Les mêmes rapports métaphoriques se tissent subtilement entre les scènes marquées, points d'articulation du récit. Les scènes de voyages et de portages de la première et de la deuxième partie du roman sous-tendent les exercices de peinture de Pierre dans la troisième partie. Cependant, et la modification est des plus significatives, le rapport analogique entre plusieurs scènes s'inverse. Par exemple, au début du récit, Pierre arrive chez Gédéon en canot par la rivière; au début de la deuxième partie du roman, Orok de son promontoire, voit le héros arriver en canot par la rivière des Mille-Tonnerre; la première rencontre humaine de la troisième partie se déroule au bord de la Seine et cette fois, c'est Pierre qui attend au bord de l'eau sans trop le savoir la venue d'un homme qu'il ne connait pas encore. La montée vers le Nord de la première partie a son pendant en troisième partie dans la tâche difficile, l'épreuve imposée à Pierre par le maître, de descendre vers le Sud. La première et la deuxième partie du roman se déroulent au rythme des déplacements géographiques du héros; dans la troisième partie, l'action se moule sur un mouvement tout intérieur du héros. En conclusion, nous pouvons dire que la première et la deuxième partie du récit sont analogues à la troisième inversée. Cette inversion dans l'agencement des scènes, donc au niveau formel, trouve son explication au niveau sémantique. La série préfigurative hétérogène qui fait état des liens unissant Pierre, entre autres au renard et au loup-cervier du Jardin des Plantes, semble fournir la raison de cette inversion. Ces bêtes que Pierre a bien connues dans ses expéditions de chasse, en première et deuxième partie du roman, il les regarde avec plaisir mais sans retrouver toutefois ce qu'il avait observé si souvent chez elles. 
Mais le regard n'était plus le même. Le regard lui échappait. Ici, le renard, dans le fond de ses propres yeux se reposait. Le loup-cervier dans le fond de ses yeux se reposait. Ces animaux au regard ni heureux ni malheureux le fascinaient. II se rappelait la torture de la faim, la frayeur de l'homme et des autres bêtes, le qui-vive constant, tout ce qui brille un instant dans un regard d'animal aperçu en forêt. A présent, dans les yeux paisibles du renard et du loup-cervier, it voyait comme un long rêve muet qui aurait cherché sa lumière ${ }^{64}$.

Dans son apprentissage de la peinture à Paris, Pierre doit surmonter plusieurs épreuves dont la première est de refouler ses souvenirs qui l'envahissent à tout propos et de "faire place nette à un présent insolite, qui ne lui paraissait pas réel. C'était comme s'il se fût appliqué à peindre un rêve flou et un peu malheureux ${ }^{65}$. "Le regard de Pierre, semblable à celui des animaux captifs, ne va plus à la rencontre du monde. II s'inverse, repose au fond des yeux, car la vision maintenant devient intérieure.

Ainsi, tout le langage du roman devient métaphorique à l'instar du mythe, cet autre langage métaphorique. La quête de la Montagne secrète se confond finalement avec un retour" aux sources profondes de la pensée et de la vie ${ }^{66}$ » qui rejoint le vieux mythe de la quête du Paradis perdu.

Marie Grenier-Francoeur

1. Philippe Hamon, "Pour un statut sémiologique du personnage", Littérature, Paris, Larousse, (mai 1972), p. 96.

2. J. J. White, Mythology in the Modern Novel, Princeton, N. J., Princeton University Press, 1971.

3. Philippe Hamon, loc. cit., p. 96.

4. Vladimir Propp, Morphologie du conte, Paris, Seuil, 1965.

5. Gabrielle Roy, la Montagne secrète, Montréal, Beauchemin, 1971, p. 12.

6. Ibid., p. 18.

7. Ibid., p. 14.

8. Le messie chrétien est une figure qui se présente comme une condensation de trois archétypes au moins: l'Enfant divin, le Voyageur au monde souterrain, le Donneur de feu.

9. La Sainte Bible, Paris, Cerf, Ex. 3, 1-7.

10. Gabrielle Roy, op. cit., p. 100.

11. Ibid. Ici et dans les autres citations c'est nous qui soulignons.

12. Ibid., p. 114.

13. Ibid., p. 108.

14. Ibid.

15. Ibid.

16. Ibid., p. 177

17. La Sainte Bible, Paris, Cerf, 1956, Gn., 32, 23-33.

18. Gabrielle Roy, op. cit., p. 103.

19. Ibid., p. 133.

20. Cf. tableau I, p. 392

21. Cf. tableau Ii, p. 393

22. Roland Barthes, Mythologies, Paris, Seuil, 1957.

23. Gabrielle Roy, op. cit., p. 219.

24. Ibid., p. 171.

25. Ibid., p. 192

26. Ibid., p. 163-165.

27. Ibid., p. 27. 
28. Ibid., p. 198.

29. Ibid., p. 203.

30. Ibid., p. 83.

31. Ibid.

32. Marcel Brion, l'Art fantastique, Paris, Marabout Université, 1968, p. 13.

33. Mircéa Éliade, Mythes et Symboles, dans l'Art fantastique, p. 13.

34. Marcel Brion, op. cit., p. 12.

35. Gabrielle Roy, op. cit., p. 84.

36. Ibid., p. 85.

37. Marcel Brion, op. cit., p. 13.

38. Gabrielle Roy, op. cit., p. 83.

39. Ibid.

40. Carl Gustav Jung, Psychology and Alchemy, dans Collected Works, vol. 12, Londres, Ruthledge and Kegan Paul, 1953.

41. Claude Levi-Strauss, Anthropologie structurale, tome 1, Paris, Plon, 1958, p. 248.

42. Gabrielle Roy, op. cit., p. 174.

43. Ibid.

44. Ibid., p. 213.

45. Ibid.

46. Ibid., p. 221.

47. Ibid., p. 158.

48. Ibid., p. 168.

49. Ibid.

50. Cité dans la Montagne secrète, p. 147.

51. Ibid., p. 74.

52. Ibid., p. 221

53. Cf. les paroles de la Montagne à Pierre, p. 102.

54. Ibid., p. 112.

55. Ibid., p. 113.

56. Gilbert Durand, les Structures anthropologiques de l'Imaginaire, Paris, Bordas, "Études supérieures", $n^{\circ}$ 14, 1969, p. 144-45.

57. Ibid., p. 196.

58. Gabrielle Roy, op. cit, p. 127.

59. Ibid.

60. Ibid., p. 173.

61. Ibid., p. 180.

62. Ibid., p. 104.

63. Ibid., p. 129.

64. Ibid., p. 181.

65. Ibid., p. 178 .

66. Ibid., p. 218. 